\title{
LA INCLUSIÓN EN LAS ENTIDADES SOCIALES. CONSTRUCCIÓN Y VALIDACIÓN DE UNA ESCALA DESDE UN MODELO MIXTO
}

\author{
Gutiérrez-Ortega, M. ${ }^{1}$, Martín-Cilleros, M.V. ${ }^{2}$ y Hernández-Soto, R. $^{3}$ \\ ${ }^{1,3}$ Universidad Internacional de la Rioja, España. ${ }^{1}$ monica.gutierrez@unir.net, ${ }^{3}$ roberto.hernandez@unir.net \\ 2Universidad de Salamanca, España. viquimc@usal.es
}

\begin{abstract}
Resumen. Introducción La inclusión es un proceso complejo que ayuda a las organizaciones a superar las barreras que limitan la participación y el aprendizaje. Las entidades sociales no cuentan en la actualidad con suficientes instrumentos que permitan conocer su posicionamiento frente a la inclusión, ni sus procesos, ni sus prácticas. Objetivos; Métodos El estudio parte de un instrumento reconocido a nivel internacional. A través de un enfoque mixto, se ha adaptado y validado para su posterior aplicación a los contextos de las entidades sociales. Se ha optado por un diseño exploratorio secuencial donde el método cuantitativo apoya al método cualitativo que es el que dirige la investigación. Resultados $A$ través de un proceso llevado en cuatro fases se desarrolla un instrumento de 24 ítems agrupados en cuatro factores, que es aplicado a 93 participantes. Conclusiones. La escala permite realizar investigaciones colaborativas y proponer actuaciones de mejora continua a partir de la propia realidad organizativa y de sus prácticas. La implementación del instrumento posibilita un tratamiento global de la diversidad en los servicios abarcando a toda la comunidad. Los métodos mixtos proporcionan un enfoque integral para abordar este tipo de estudios.
\end{abstract}

Palabras clave: Cultura Inclusiva; Prácticas Inclusivas; Participación; Comunidad; Método Mixto

\section{INCLUSION IN SOCIAL ENTITIES. CONSTRUCTION AND VALIDATION OF A SCALE FROM A} MIXED MODEL

Abstract. Introduction Inclusion is a complex process that helps organizations overcome barriers that limit participation and learning. Social entities do not currently have instruments that allow them to know their position regarding inclusion, their processes, or their practices. Goals; Methods The study builds on an internationally recognized instrument. Through a mixed approach, it has been adapted and validated for its subsequent application to the contexts of social entities. We follow a sequential exploratory design in which quantitative methods complement the qualitative approach that sustains the investigation. Results Through a process carried out in four phases, a 24-item instrument grouped into four factors was developed and utilized with a sample of 93 participants. Conclusions. The scale allows collaborative research and proposes continuous improvement actions based on organizational reality and its practices. The implementation of the instrument allows a global treatment of diversity in services covering the entire community. Mixed methods provide a comprehensive approach to address these types of studies.

Keywords: Inclusive Culture; Inclusive Practices; Participation; Community; Mixed Method

\section{INTRODUCCIÓN}

La inclusión es un proceso complejo que se puede conceptualizar en múltiples niveles, incluyendo al individuo, los grupos, las organizaciones y el sistema social (Barrio de la Puente, 2009). La UNESCO definió la inclusión como un proceso que ayuda a superar las barreras que limitan la presencia, participación y logro de las personas. (UNESCO, 2017; Gutiérrez-Ortega, Martín-Cilleros \& Jenaro-Rio, 2018). 
Como proceso que es, no se puede analizar de forma estática puesto que se crea de nuevo en cada situación a través de la relación de las personas con su entorno social (Ferdman \& Davidson, 2002). La inclusión, por tanto, implica un conjunto dinámico e interrelacionado de procesos.

Al mismo tiempo, los sentimientos de inclusión, o el comportamiento y la actitud inclusiva que se pueden experimentar en un momento dado pueden no significar demasiado si no vienen arropados por una historia, una cultura, una comunidad, una estructura y un sistema que les dé el significado apropiado (Ferdman \& Davidson, 2002; Ferdman, 2017). En este sentido, se puede decir que la inclusión es una práctica: un conjunto de estructuras que interactúan, valores, normas, climas grupales y organizacionales, y comportamientos colectivos, que hace que todas las personas involucradas estén conectadas con la experiencia de inclusión en un sistema dinámico y de refuerzo mutuo.

Las entidades sociales no cuentan en la actualidad con suficientes instrumentos que permitan conocer su posicionamiento frente a la inclusión, ni sus procesos, ni sus prácticas. Por ello, desde la presente investigación, se parte de un instrumento, afianzado a nivel internacional (Booth y Ainscow, 2002, 2011), para a través de un enfoque mixto, adaptarlo y validarlo para su posterior aplicación a los contextos de las entidades sociales. Las preguntas de investigación que nos planteamos son:

- ¿Puede adaptarse el índex para la inclusión para su aplicación en entidades sociales?

- ¿Puede crearse una escala de evaluación que ayude a los centros a evaluar la inclusión entre sus miembros?

- ¿Una metodología mixta de investigación puede apoyar los procesos de adaptación y validación de una escala de evaluación como la que se presenta?

\subsection{Inclusión}

La inclusión como se ha señalado es un proceso complejo que requiere una mirada que se aleje de un enfoque simplista que puede ocultar muchas sutilezas y matices $y$, no tiene en cuenta aspectos relevantes que se viven en los contextos donde abunda la diversidad (McMaster, 2015). Otro aspecto clave de la complejidad del concepto tiene que ver con el marco de referencia para definir qué constituye la inclusión. Independientemente de que se hable de una persona o una organización, ¿qué es una práctica organizativa inclusiva o un comportamiento individual inclusivo? 
Según Ferdman y Deane (2014) la inclusión, en cualquiera de los niveles debe basarse en si los afectados por la práctica o por el comportamiento se sienten incluidos y en si, al mismo tiempo, lo están. Es decir, la inclusión debe ser conceptualizada y debe estar asociada siempre a las percepciones e interpretaciones de las personas. Un conjunto de hechos objetivos no necesariamente puede determinar si existe inclusión; deben ser evaluados en base a la experiencia de los involucrados, por lo tanto, podrían variar de una persona a otra y de una situación a otra (Azorin \& Ainscow, 2018).

\subsection{Index para la inclusión}

El Index para la inclusión fue desarrollado por el Centro de Estudios sobre Educación Inclusiva (Booth \& Ainscow, 2002, 2011). La UNESCO (2002) lo definió como un conjunto de materiales diseñados para apoyar a las escuelas con sus procesos y avanzar hacia la educación inclusiva. Instrumentos como el Index para la inclusión pueden ayudar a las organizaciones a poner en marcha un proceso de autoevaluación (de las tres dimensiones claves en la inclusión como son la cultura, las políticas y las prácticas. Facilitando de este modo el desarrollo de procesos de planificación y colaboración, acordes a los valores y contextos, que sean sostenibles en el tiempo (Rustemier \& Booth, 2005). El instrumento se articula a través de una estructura jerárquica que, partiendo de las tres dimensiones señaladas, va especificándose en secciones, indicadores y preguntas (Figura 1).

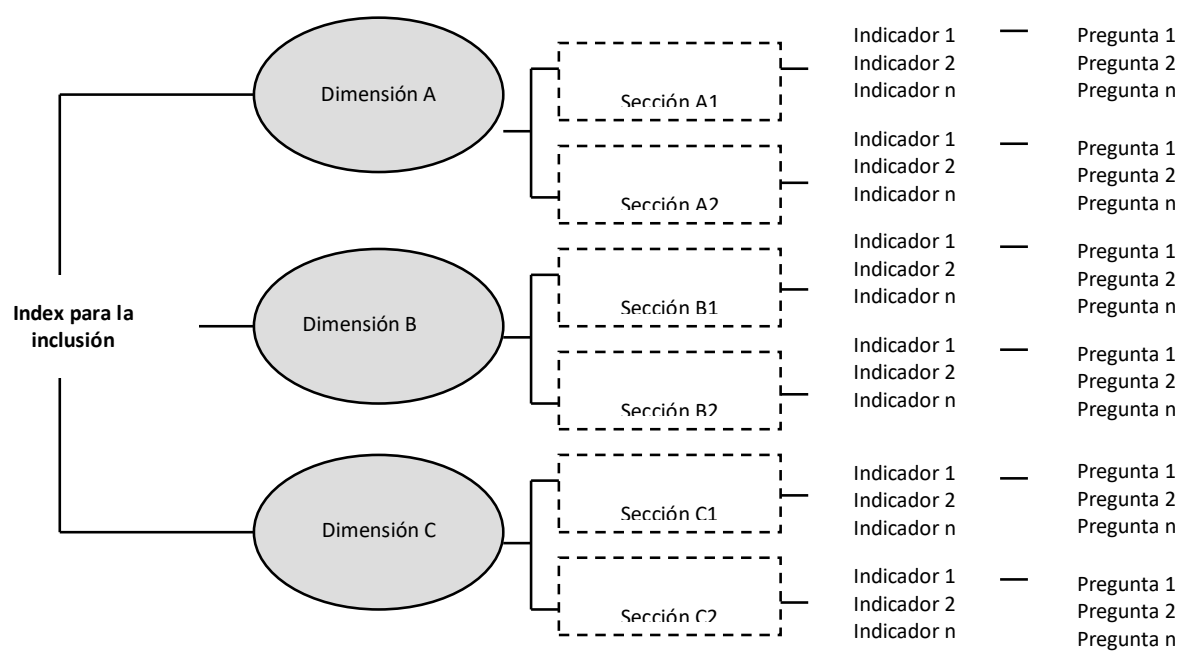

Figura 1. Esquema del Index para la Inclusión (Gutiérrez-Ortega, Martín-Cilleros, Jenaro-Rio, 2014)

\section{DISEÑO DE LA INVESTIGACIÓN}


Por la complejidad del constructo a evaluar se ha diseñado una investigación que combina de enfoques cualitativos y cuantitativos para abordar la temática que nos ocupa de una forma holística y más completa (Creswell, 2014; Creswell et al., 2004). Y, para que la riqueza de los datos sea superior ya que las fuentes son diversas (Brannen, 1992). Siguiendo a Tashakkori \& Teddlie (2003, p. 11) adoptamos un modelo de investigación mixto porque en las diferentes fases tendrán presencia los diferentes enfoques.

Se ha optado por un diseño exploratorio secuencial donde el método cuantitativo apoyará al método cualitativo que es el que marcará la investigación (Greene \& Caracelli, 2003). Por ello, la investigación se iniciará con la obtención de datos cualitativos para explorar la inclusión en la entidad seleccionada, y posteriormente se llevará a cabo una etapa cuantitativa de la cual se extraerán resultados que se conectarán con los obtenidos en la etapa inicial.

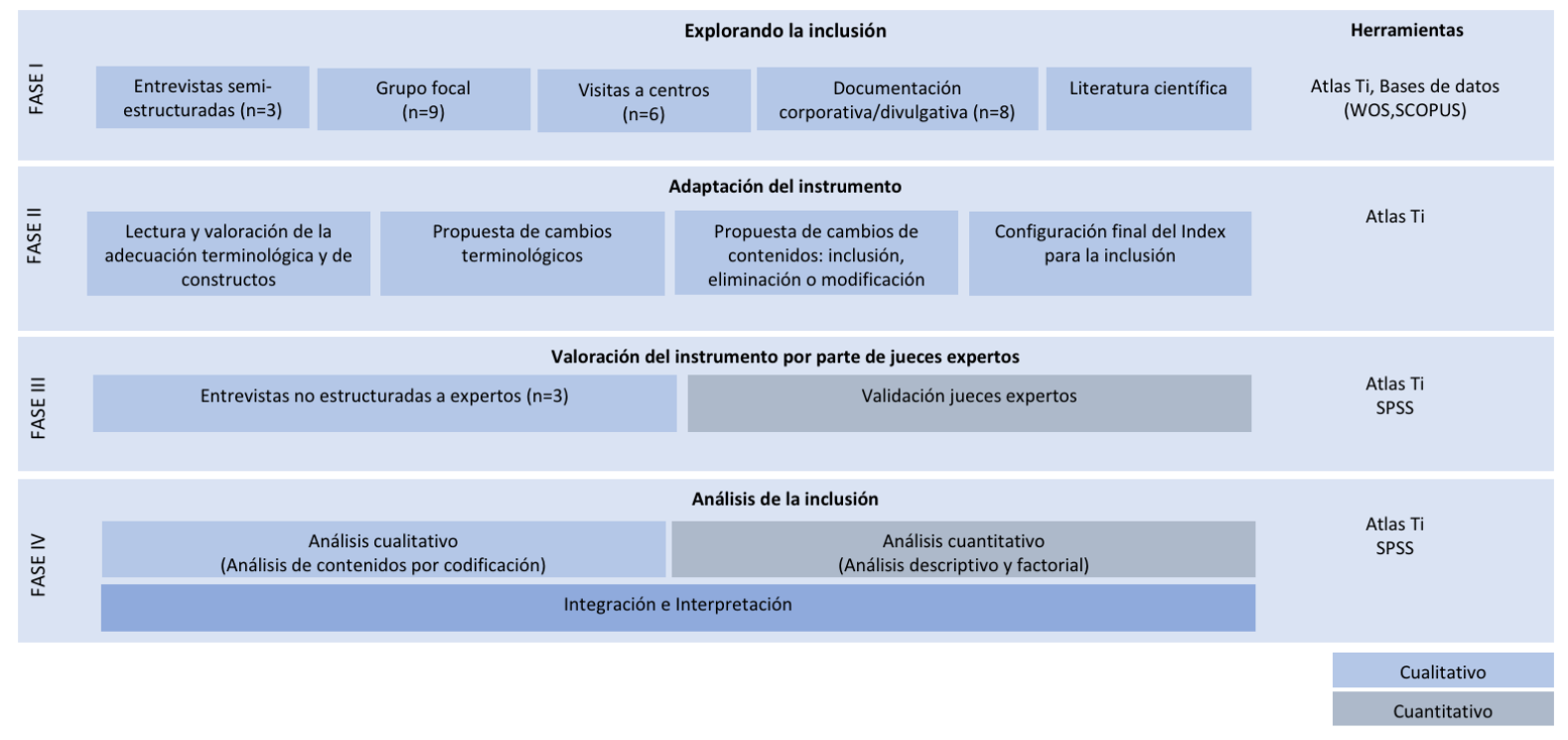

Figura 2. Diseño de la investigación.

La adaptación del instrumento se ha realizado tomando como punto de partida una entidad social de referencia a nivel internacional por su perspectiva y planteamientos ante la inclusión. A continuación, se detallan las fases llevadas a cabo.

\subsection{Fase 1. Explorando la inclusión}

Se realizaron tres entrevistas semi-estructuradas con miembros del equipo directivo y posteriormente, un grupo focal con una muestra intencional formada por tres directores de 
centros y seis técnicos responsables. El propósito del grupo focal fue generar ideas sobre áreas críticas y/o problemas relacionados con la cultura, las políticas y las prácticas inclusivas en el centro.

Después de la realización de grupo focal se realizaron un total de 6 visitas a centros de la entidad para recoger la opinión de los técnicos y de las personas usuarias.

De forma complementaria se analizó documentación de la entidad tanto corporativa como divulgativa (por ejemplo, revistas o los libros publicados por la organización). El objetivo fue analizar el discurso que se realizaba sobre la inclusión.

Los investigadores $(n=3)$ tras la codificación de las entrevistas, la sesión de grupo focal y el análisis documental en Atlas Ti 8.0, extrajeron un total de seis familias (comunidad, valores, centro compartido, organización de la actividad, dirección, recursos) (Braun \& Clarke, 2006) que marcaron la revisión y adaptación del Index for Inclusion.

\subsection{Fase 2. Adaptación del Index para la Inclusión}

El proceso de adaptación del Index se llevó a cabo en toda su amplitud por lo que se adaptaron dimensiones, secciones, indicadores y preguntas. Los cambios se realizaron teniendo en cuenta los datos codificados en la etapa anterior.

Tabla 1. Cambios realizados en el Index para la inclusión

\begin{tabular}{lllllr}
\hline & Mantenido & Adaptado & Modificado & Eliminado & Añadido \\
\hline Dimensiones & 3 & 0 & 0 & 0 & 0 \\
Secciones & 1 & 3 & 2 & 0 & 0 \\
Indicadores & 2 & 30 & 8 & 4 & 2 \\
Preguntas & 46 & 335 & 67 & 74 & 27
\end{tabular}

El proceso constó de cuatro etapas: (1) Lectura y valoración de la adecuación terminológica y de constructo, (2) Propuesta de cambios terminológicos vinculándolos a los temas y comentarios extraídos de las entrevistas y grupo focal (codificados en Atlas $\mathrm{TI}$ en las seis familias ya indicadas), (3) Propuesta de cambios de contenidos: inclusión, eliminación o modificación en su caso, y para finalizar (4) Configuración final del Index para la inclusión para entidades sociales.

Se ilustra con unos ejemplos en la tabla 2 como se ha pasado de los textos codificados a los cambios en indicadores y preguntas. 
Tabla 2. Relación entre los datos obtenidos en Fase I y la adaptación realizada en Fase II.

\begin{tabular}{|c|c|c|}
\hline Cita & Indicador & Pregunta \\
\hline $\begin{array}{l}\text { "...hemos desarrollado una } \\
\text { amplia cultura de alianzas, } \\
\text { entendiendo la cooperación } \\
\text { institucional como base para el } \\
\text { desarrollo". Entrevista Equipo } \\
\text { Directivo (ED_01) }\end{array}$ & $\begin{array}{l}\text { Todas las instituciones } \\
\text { de la comunidad están } \\
\text { involucradas en el } \\
\text { centro. }\end{array}$ & $\begin{array}{l}\text { ¿El centro desarrolla una cultura de } \\
\text { alianzas, entendiendo la cooperación } \\
\text { institucional como base para el } \\
\text { desarrollo? }\end{array}$ \\
\hline $\begin{array}{l}\text { "Si trabajamos permanentemente } \\
\text { por el progreso de cada persona, } \\
\text { por grave que sea su } \\
\text { discapacidad, veremos con } \\
\text { frecuencia como sus limitaciones } \\
\text { aminoran". Cita documental } \\
\text { (DO_03) }\end{array}$ & $\begin{array}{l}\text { Se valora la capacidad } \\
\text { de todas las personas } \\
\text { para desarrollar su } \\
\text { potencial. }\end{array}$ & $\begin{array}{l}\text { ¿En el centro se cree que, si se } \\
\text { trabaja permanentemente por el } \\
\text { progreso de cada persona, por grave } \\
\text { que sea su discapacidad, sus } \\
\text { limitaciones se aminoran? }\end{array}$ \\
\hline $\begin{array}{l}\text { "La motivación, el empeño, la } \\
\text { tenacidad son vitales para lograr } \\
\text { progresos y mayor grado de } \\
\text { autonomía". (GF) }\end{array}$ & $\begin{array}{l}\text { Se implica activamente } \\
\text { a todas las personas } \\
\text { usuarias en su propio } \\
\text { aprendizaje. }\end{array}$ & $\begin{array}{l}\text { ¿Se cree que la motivación, el } \\
\text { empeño, la tenacidad, son vitales } \\
\text { para lograr progresos y mayor grado } \\
\text { de autonomía? }\end{array}$ \\
\hline
\end{tabular}

Una vez adaptado el Index para la inclusión, se seleccionaron sus indicadores y con ellos se construyó la Escala de Evaluación de la Inclusión (EEI). A continuación, se procedió a valorar la pertinencia o intensidad, es decir, adecuación de cada ítem para medir los diferentes constructos o conceptos incluidos en el Index, así como la dimensión a la que podría pertenecer cada uno de los ítems seleccionados. La escala elaborada contiene un total de 42 ítems que se corresponden con los indicadores del Index adaptado a las entidades sociales.

\subsection{Fase 3. Valoración del instrumento por parte de jueces expertos}

La escala fue inicialmente valorada a través de tres entrevistas no estructuradas por jueces expertos de la entidad con el objetivo de recoger opiniones sobre la redacción y presentación de la escala.

Obtenidos los datos, se procedió a modificar la escala para adecuarlo a las características los usuarios finales. Así se ajustaron las descripciones de las secciones y, por otra parte, se incluyeron elementos de apoyo en los indicadores para que fueran accesibles a personas con cualquier tipo de limitación. Como apoyo se optó por seleccionar para cada ítem dos de las preguntas más representativas.

El siguiente paso consistió en el análisis cuantitativo, a través de la escala, de la fiabilidad del instrumento por parte de jueces externos $(n=14)$ cuidando la representación de los diferentes perfiles que se pueden encontrar en los centros, directivos, profesionales, familias 
y personas usuarias. Los índices de concordancia fueron bajos lo que conllevó a la elaboración de una segunda versión de la escala preliminar $(n=40)$ empleando el acuerdo inter-jueces para la eliminación de los ítems que generaban confusión. Se siguieron para ello las directrices de Lombard, Snyder-Duch y Bracken $(2002,2003)$ y los datos obtenidos en la fase cualitativa de la validación de jueces.

\subsection{Fase 4. Análisis de la inclusión}

Los análisis cualitativos se realizaron en primer lugar para conocer el fenómeno en la entidad seleccionada. Se analizaron en profundidad los textos transcritos extraídos en las fases anteriores a través de las entrevistas y el grupo focal.

En la fase cuantitativa, una vez definida la escala en la fase anterior se seleccionó una muestra intencionada de 93 personas pertenecientes a la entidad. La muestra representa a todos los colectivos que forman parte de la organización. Se realizó el análisis de los datos de la valoración de la escala a través del SPSS versión 21.0.

El análisis de la consistencia interna se realizó calculando el coeficiente alfa de Cronbach y la evaluación de la validez de constructo se ejecutó por medio del análisis factorial exploratorio: análisis de componentes principales con rotación Oblimin, por tratarse de factores relacionados, seguido de un análisis que empleó rotación Varimax para tratar de extraer componentes lo más independientes posible.

El análisis puso de manifiesto la existencia de algunos ítems que saturaban con claridad en un factor, mientras que otros ítems saturaban en varios factores. Estos últimos fueron eliminados, lo que permitió finalmente obtener una escala definitiva, compuesta por cuatro factores y un total de 24 ítems que explican el $56 \%$ de la varianza.

En la integración de los datos se puede ver coinciden los datos de ambas fases. El aspecto que llama más la atención es la importancia que toda la organización da a los Valores y a la Comunidad. En la fase cuantitativa se desdoblan los datos en dos factores, y no de forma conjunta como lo hace el Index, y en la fase cualitativa son múltiples las citas asociadas a estos temas: [GF: “...muchas personas nos comentan, después de visitar nuestros, que sorprende escuchar a los profesionales, a las familias, y los chicos y el mensaje de todos es el mismo. Todos hablamos con entusiasmo del proyecto"] [ED03: "nos hemos construido sobre nuestros valores]. 


\section{CONCLUSIONES}

Los hallazgos de este estudio ofrecen respuestas a las preguntas que se planteaban al inicio de la investigación. En primer lugar, podemos señalar que el proceso realizado y los resultados obtenidos muestran que un instrumento como el Index para la inclusión diseñado para contextos educativos puede adaptarse para entidades sociales. Tras los análisis realizados de las entrevistas y grupo focal unido a los datos cuantitativos obtenidos podemos ver que hay validez de contenido y de constructo. Por lo que podemos concluir diciendo que los cambios que se han realizado en las secciones del Index han resultado adecuados y reflejan la realidad de una entidad social. Si analizamos los resultados obtenidos en el análisis factorial podemos ver cómo los cuatro factores que se han obtenido son los mismos que los que presenta el Index para la Inclusión para centros escolares, aunque es de destacar que la Dimensión Cultura Inclusiva se desdobla en dos factores: comunidad y valores. Este aspecto es coherente con los datos extraídos en el análisis de contenido realizado de las entrevistas, grupo focal y revisión de la documentación de la organización. Otro aspecto que destacar es que en cada uno de los factores se entremezclan los indicadores del Index para la inclusión, lo cual demuestra que las tres dimensiones no son espacios estancos y que, en la vida diaria de los centros, lejos de los aspectos teóricos, las influencias son múltiples y muy variadas. Este aspecto fue el tema más debatido y que, como se ha señalado, llevó a la adaptación de categorías y su redefinición.

En segundo lugar, si nos centramos en la metodología aplicada se puede concluir se ha demostrado que un método como el empleado nos permite identificar factores que están bajo conductas organizativas y humanas, y que las técnicas cualitativas y cuantitativas utilizadas han dado luz sobre muchos aspectos que se hubieran quedado ocultos si sólo se hubiera utilizado un enfoque cuantitativo. La combinación de múltiples técnicas en las diferentes etapas de la investigación ha hecho que obtengamos datos más ricos y sólidos.

La replicación de este estudio en otras entidades sociales proporcionará más evidencia de la generalización del instrumento.

\section{REFERENCIAS}

Azorín, C.M. \& Ainscow, M. (2018). Guiding schools on their journey towards inclusion. International Journal of Inclusive Education,24(1), 58-76. https://doi.org/10.1080/13603116.2018.1450900

Barrio de la Puente, J. L. (2009). Hacia una Educación Inclusiva para todos. Revista Complutense de Educación, 
$20(1), 13-31$

Booth, T., \& M. Ainscow. 2002. Index for Inclusion: Developing Learning and Participation in Schools. Bristol: Centre for Studies on Inclusive Education.

Booth, T. \& Ainscow, M. (2011). Index for Inclusion: developing learning and participation in schools. Bristol: Centre for Studies in Inclusive Education (CSIE).

Brannen, J. (Ed.). (1992). Mixing methods: Qualitative and quantitative research. Aldershot: Avebury.

Braun, V., \& Clarke, V. (2006). Using thematic analysis in psychology. Qualitative research in psychology, 3(2), 77-101.

Creswell J.W., Fetters M.D. \& Ivankova N.V. (2004). Designing a mixed methods study in primary care. Ann Fam Med, 2,7-12.

Creswell, J.W. (2014). Research design: qualitative, quantitative, and mixed methods approaches. Thousand Oaks, CA: Sage.

Ferdman, B. M. (2017). Paradoxes of inclusion: Understanding and managing the tensions of diversity and multiculturalism. The Journal of Applied Behavioral Science, 53(2), 235-263. https://doi.org/10.1177/0021886317702608

Ferdman, B. M. \& Davidson, M. N. (2002). A matter of difference-Inclusion: What can I and my organization do about it? The Industrial-Organizational Psychologist, 39(4), 80- 85.

Ferdman, B. M. \& Deane, B. R. (Eds.). (2014). Diversity at work: The practice of inclusion. San Francisco, CA: Jossey-Bass. https://doi.org/10.1002/9781118764282.ch1

Greene, J. C., \& Caracelli, V. J. (2003). Making paradigmatic sense of Mixed Methods practice. En A. Tashakkori \& C. Teddlie (Eds.), Handbook Mixed Methods in social and behavioral research (pp. 91- 110). Thousand Oaks, CA: Sage.

Gutiérrez-Ortega, M., Martín-Cilleros, M. V. \& Jenaro, C. (2014). El Index para la inclusión: presencia, aprendizaje y participación. Revista de Educación Inclusiva, 7(3), 186-201. http://www.revistaeducacioninclusiva.es/index.php/REl/article/view/139/133

Gutiérrez-Ortega, M., Martín-Cilleros, M. V. \& Jenaro, C. (2018). La cultura, pieza clave para avanzar en la inclusión en los centros educativos. Revista de Educación Inclusiva, 11(2), 13-26.

Lombard, M., Snyder-Duch, J., \& Bracken, C. C. (2002). Content analysis in mass communication: Assessment and reporting of intercoder reliability. Human Communication Research, 28, 587-604.

Lombard, M., Snyder-Duch, J., \& Bracken, C. C. (2003). Correction. Human Communication Research, 29, 469472

McMaster, Ch. (2015). Inclusion in New Zealand: The Potential and Possibilities of Sustainable Inclusive Change Through Utilising a Framework for Whole School Development. New Zealand Journal of Educational Studies, 50(2), p239-253. doi: 10.1007/s40841-015-0010-3

Rustemier, S., \& Booth, T. (2005). Learning about the Index in use; a study of the Index for Inclusion in schools and LEAs in England. Bristol CSIE.

Tashakkori, A., \& Teddlie, C. (2003). Handbook of mixed methods in the social and behavioral sciences. Thousand Oaks, CA: Sage.

UNESCO (2017). A Guide for Ensuring Inclusion and Equity in Education. Paris: UNESCO.2002

Watts, M., \& Alsop, S. A. (2000). Terms of Engagement: Learners and School Science. In Paper presented to the 
Annual Conference of the Canadian Society for the Study of Education. University of Edmonton, Canada. 